\title{
Practical implications of uncertainty in observed SSTs
}

\author{
Lisa Goddard, ${ }^{1}$ David G. DeWitt, ${ }^{1}$ and Richard W. Reynolds ${ }^{2}$ \\ Received 10 February 2009; revised 30 March 2009; accepted 10 April 2009; published 13 May 2009.
}

[1] Uncertainties in the accuracy of observed sea surface temperature (SST) estimates limit a number of efforts relevant to seasonal-to-interannual climate variability and its prediction. Some of the efforts that may be hampered by uncertain SSTs include estimates of skill in predicted SSTs, attribution studies of seasonal climate anomalies, and calibration of probabilistic seasonal climate forecast systems. This study examines the explicit impact of SST uncertainties on the climate response from an atmospheric general circulation model. Uncertainties in Western Pacific SSTs play a substantial role in the sensitivity of the seasonal climate. Citation: Goddard, L., D. G. DeWitt, and R. W. Reynolds (2009), Practical implications of uncertainty in observed SSTs, Geophys. Res. Lett., 36, L09710, doi:10.1029/ 2009GL037703.

\section{Introduction}

[2] Observations are not perfect. Errors can arise from the instruments, from their reporting, and from analyses, particularly gridded analyses in regions of sparse measurements. Uncertainty in monthly gridded SST fields comes primarily from sampling errors [Smith and Reynolds, 2005]. Errors in SSTs have implications not only for their own variability and trends, but also for interpreting their impact on the overlying atmosphere and subsequently on terrestrial climate. Uncertainties in SSTs become particularly relevant through their use in driving and validating dynamical models used for seasonal climate prediction and attribution, addressing questions such as: "What was the relative effect of SST anomalies in the western Pacific on United States winter temperatures last year?"

[3] One goal of the Sustained Ocean Observing System for Climate is to improve the accuracy of SST observations over the global ocean. The targeted performance for observed SSTs, for 2008, is accuracy of at least $0.4^{\circ} \mathrm{C}$ [cf. Office of Climate Observations, 2008, p. 46] on a $5^{\circ}$ latitude-longitude grid. A single number applies to all grid points over the global ocean. The actual errors and their relative importance vary greatly in space and time. Some of the regions with the largest estimated SST uncertainty, such as the western Pacific and Indian Oceans exhibit the lowest interannual variance. These areas have not been well sampled with in-situ data. Also, the mean SST in these regions typically is high enough to sustain deep convection, which limits the ability of satellites to measure SSTs. Because of the importance of both the absolute magnitude

\footnotetext{
${ }^{1}$ International Research Institute for Climate and Society, Earth Institute at Columbia University, Palisades, New York, USA.

${ }^{2}$ National Climate Data Center, NOAA, Camp Springs, Maryland, USA.

Copyright 2009 by the American Geophysical Union. 0094-8276/09/2009GL037703
}

of SST and the regional SST gradients over these regions, seemingly small errors in local SSTs could have important implications for dynamical climate prediction.

[4] This study illustrates some potential implications of uncertain SST observations for seasonal climate simulations and predictions by explicitly incorporating observational error estimates into the boundary conditions of atmospheric general circulation model (AGCM) experiments. Hoerling and Kumar [1997] examined the sensitivity of several AGCM simulations to observed SST analyses that differed by up to $1.5^{\circ} \mathrm{C}$ in the Niño3.4 region during 1982-83, and noted that only with the larger reanalysis values did the magnitude of the models' forced response become similar to that observed. This study examines the sensitivity to much smaller errors in the observed SST estimates.

[5] The results highlight the need to consider uncertainty in SSTs for validating seasonal predictions of tropical SSTs and for validating and providing associated probabilistic seasonal climate forecasts. This work additionally suggests the societal benefit possible by reducing gaps in the global ocean observing network, arising from improved ability to monitor and predict the climate system.

\section{Data and Experimental Design}

[6] The observed SST data, and its associated uncertainties, come from the Extended Reconstructed SST data set version 2 (ERSSTV2) [Smith and Reynolds, 2005]. This data provides monthly data for the period 1854-present on a $2 \times 2^{\circ}$ horizontal grid. The uncertainty, or error, comes from three possible independent sources: bias, sampling and random error. Random error is typically smaller than bias and sampling errors on monthly time scales. A rough estimate of the global in situ bias error can be obtained by comparing buoy and ship data. Collocated monthly differences for an 18 year period (1989-2006) show that ships are warmer than buoys by $0.13^{\circ} \mathrm{C}$. The standard deviation of monthly sampling error [Smith and Reynolds, 2004] ranges between 0.2 and $1^{\circ} \mathrm{C}$ after 1970 over most of the ocean and dominates the other two types of errors.

[7] The SST data are employed in two complementary analyses of relevance to seasonal climate prediction. As it is the lower boundary conditions, principally SSTs, that drive seasonal-to-interannual climate variability, predicting climate involves not only predicting the response to changing patterns of SSTs, but also predicting the SSTs themselves. The first analysis examines the presumed upper limit of skill for predicting SSTs, based on uncertainty in the "true" value. The second analysis examines the sensitivity of the climate system to the sign and magnitude of estimated error in select case studies, as estimated by an AGCM.

[8] The AGCM employed here is the ECHAM4.5 configured at T42 with 19 vertical layers [Roeckner et al., 1996]. For the period 1950-2003, 20 ensemble members 
(a) <Correlation> : Fixed Error

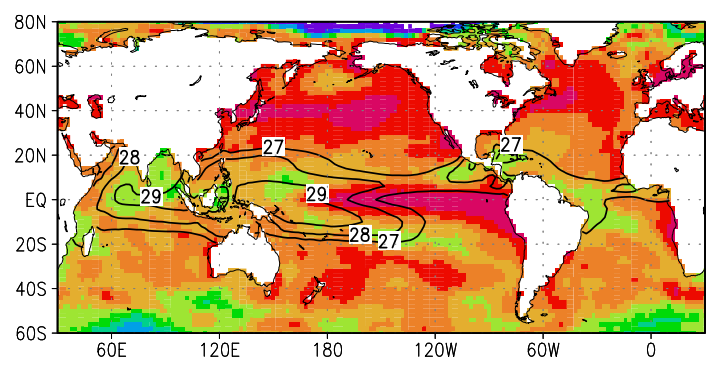

(b) MASKED <Corr> : Sampling Error

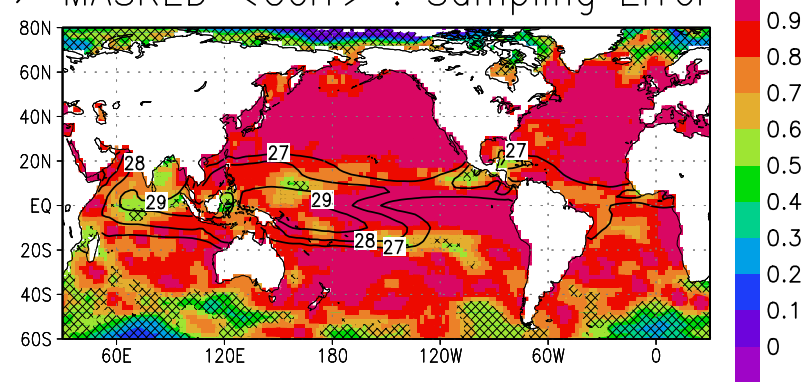

(c) Interannual Variance of SSTa

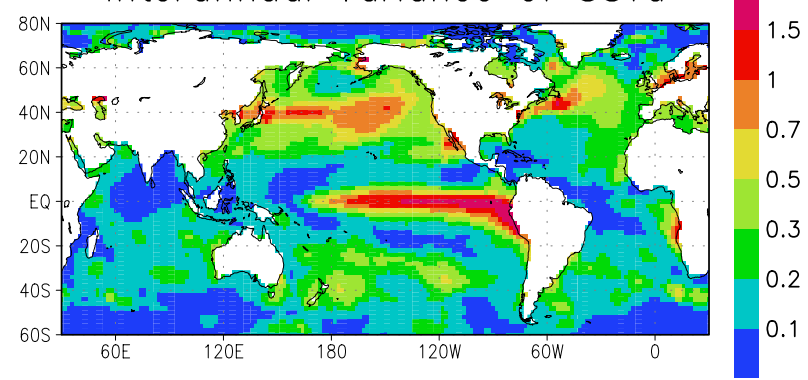

Figure 1. Correlation between monthly observed time series, taken as "truth", and that with random Gaussian error over the period 1983-2004. (a) Error magnitude assumed fixed at $0.4^{\circ} \mathrm{C}$. (b) Varying error magnitude taken from estimated sampling error variance of the ERSSTv2 data. Hatched regions delimit where the expected correlation exceeds that in Figure 1a. (c) Interannual variance of monthly mean SST anomalies. Superposed black contour indicates climatological SST at $27,28,29^{\circ} \mathrm{C}$, demarking the warmest and most convective regions of the tropics.

were generated with ERSSTv2 SSTs imposed over the global oceans. The ensemble members differ from each other only by the realization of the model weather at the start of the integrations. This ensemble size suffices to accurately estimate the ensemble mean for seasonal temperature and precipitation in this model; larger ensemble sizes lead to only small incremental changes in local anomaly correlation coefficients, for example. For the analysis examining sensitivity of the seasonal climate to SST uncertainty, two sets of experiments were conducted. The first applies the observational error estimate to the global oceans. The second applies the observational error estimate only over the western Pacific where the in-situ data is sparse and remotely sensed data is more problematic. Three cases were chosen for these experiments, and the estimated standard error was added and subtracted from the "true" SST value. Looking at just the January-March season, the three cases are: 1983 - El Niño conditions, 1986 - ENSO-neutral conditions, and 1989 - La Niña conditions. For these 12 experimental runs ( 2 choices of spatial domain for error, 2 signs of error, and 3 JFM seasons), 20 ensemble members were generated, taking their initial conditions from the appropriate point in the history files of the long integrations with the "true" SSTs.

\section{Results}

\subsection{SST Potential Predictability Estimates}

[9] Figures $1 \mathrm{a}$ and $1 \mathrm{~b}$ show the predictability limit, as measured by expected correlation, for the ERSSTv2 data. Monte Carlo sampling is used to generate 1000 correlation fields by correlating the "true" value with the same added to a randomly generated number drawn from a Gaussian distribution with a standard deviation given by the observational uncertainty. The median value is plotted. In Figure 1a, a fixed error standard deviation, or uncertainty, is assumed of $0.4^{\circ} \mathrm{C}$. This value is extracted as a rough estimate of the globally-averaged RMS differences between several analyses for the 1982-97 period as shown in Figure 11 of Smith and Reynolds [2004]. Low correlation values reflect low interannual variability (Figure 1c), such that magnitude of the error is more comparable to the variability. In calculating the expected correlation of Figure $1 \mathrm{~b}$, a spatially and temporally varying error is given by the sampling error standard deviation estimated for the ERSSTV2 data. The areas of relatively low correlation reflect low variability and/or high sampling error. Hatched regions in Figure $1 \mathrm{~b}$ indicates where the $0.4^{\circ} \mathrm{C}$ target is not being met as the expected correlation, which relates to the expected uncertainty, is greater than that in Figure 1a. Within the temperate and tropical latitudes, the regions where the uncertainty is greater than or equal to $0.4^{\circ} \mathrm{C}$ are the convective regions of the western Pacific, Indian Ocean, northeast Pacific warm pool, and the south Pacific convergence zone.

[10] The proximity of the low skill areas to the warm pool and other convergence regions (shown by heavy black contours on Figure 1) in part explains their existence. Where the SST is relatively warm, low-level convergence and convection exists in the vicinity. The clouds resulting from the convection hamper the infrared satellites from accurately measuring SST. Satellite data does not contribute directly to the ERSST data, but it is used to determine the spatial SST modes used to reconstruct the monthly fields from the available in situ SST data. The implications of the uncertain SST regions for simulating climate with AGCMs is that an incorrect representation of changes in these fringe areas of the warmest SST regions can adversely affect the spatial representation of SST anomalies, altering the strength and location of convection and associated upper and lower tropospheric flow.

[11] A related point is that SST skill maps from coupled GCMs (CGCMs, not shown) are often roughly consistent with the regions of lower potential predictability or verifiability. This suggests that an upper limit of skill should be something similar to what is shown in Figure 1b. It could be argued that SST predictions should not be expected to exceed these values and that the skill of SST predictions 
(a) JFM $1983 \mathrm{SSTa}$

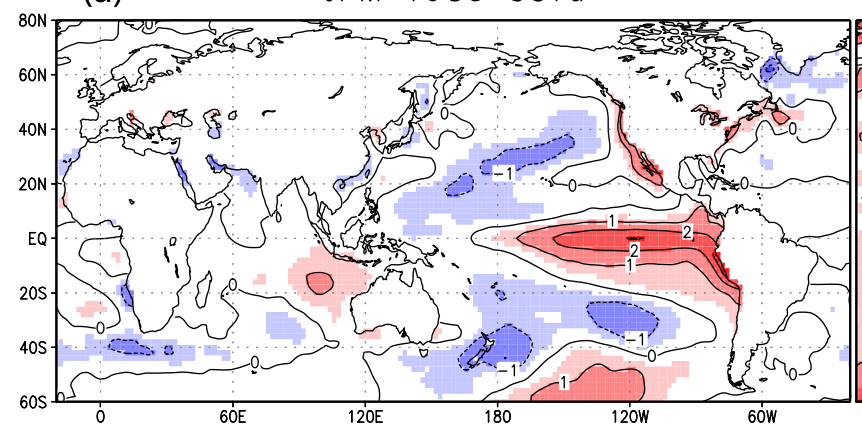

(b)

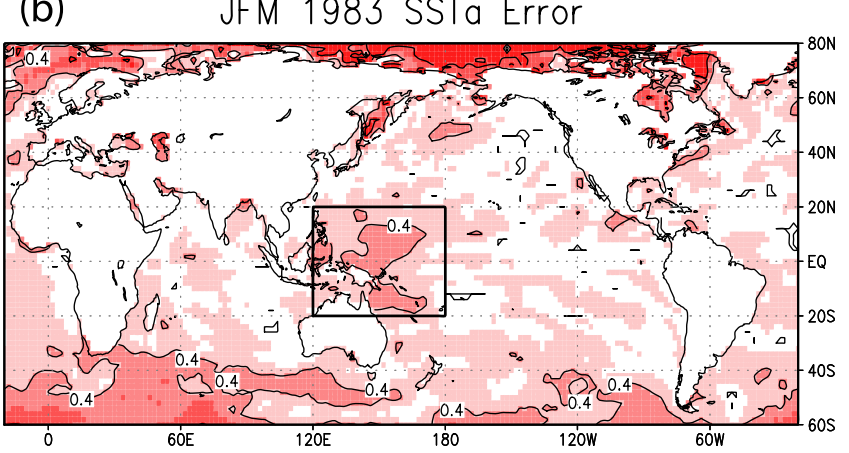

(c)

JFM 1986 SSTa

(d)

JFM 1986 SSTa Error

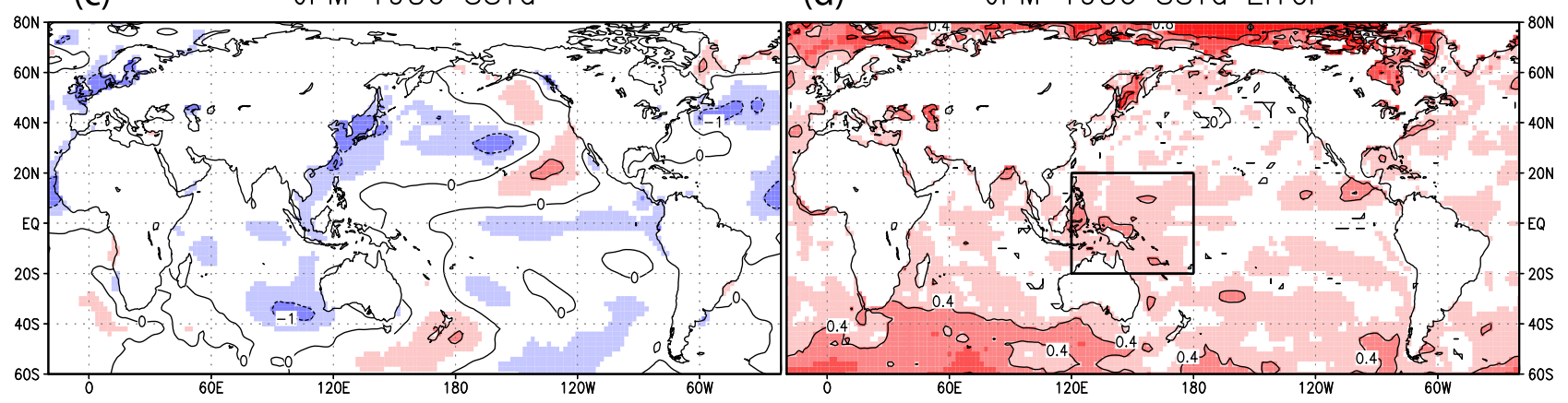

(e)

JFM 1989 SSTa

(f)

JFM 1989 SSTa Error

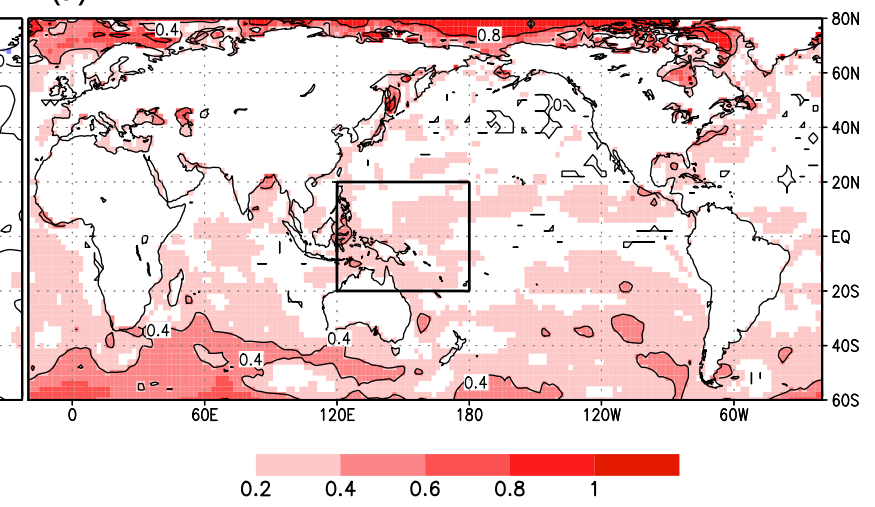

Figure 2. (a, c, and e) Sea surface temperature anomalies $\left({ }^{\circ} \mathrm{C}\right)$, relative to the 1983-2002 climatology for the three sensitivity cases, (b, d, and f) together with their associated error estimates. The box in Figures 2a, 2c, and 2e delimits the 'Western Pacific' region $(20 \mathrm{~S}-20 \mathrm{~N} ; 120 \mathrm{~W}-180 \mathrm{~W})$ used in the sensitivity experiments.

should be presented relative to such an upper limit that explicitly accounts for the uncertainties in the verification data. This is the first practical implication of uncertainties in observed SSTs.

\subsection{Sensitivity of Seasonal Climate}

[12] The sensitivity of the seasonal climate response of an AGCM is examined for three case studies using the JanuaryFebruary-March season with El Niño conditions (Figure 2a), ENSO-neutral conditions (Figure 2c), and La Niña conditions (Figure 2e). SST anomalies, and subsequent climate anomalies, are defined relative to the 20 -year climatology period of 1983-2002. Two sets of experiments add and subtract, in turn, the estimated seasonal-mean error standard deviation (Figures 2b, 2d, and 2f) to the observed SST fields. The first set of experiments applies the error field to the global oceans. The second set of experiments, applies the error field only over the western Pacific box $(20 \mathrm{~S}-20 \mathrm{~N}$; $120 \mathrm{~W}-180 \mathrm{~W})$.

[13] The sensitivity analysis provides an estimate of the impact of stated errors on the seasonal climate response of an AGCM. In all cases examined, the SST errors lead to a discernible modification of temperature and precipitation anomalies globally (Figure 3). Significance, assessed by t-test, is exaggerated over the ocean, since SSTs are prescribed in AGCM experiments, and thus the ensemble variance is near zero. In the first set of experiments, where the error field is applied over the global ocean, it is difficult to discern how much of the temperature response is driven by errors in the tropics and how much is due to fact that the global oceans are slightly cooler/warmer than "observed" (e.g., Figures $3 \mathrm{c}$ and $3 \mathrm{~g}$ ). In the second set of experiments, where the error field is applied only over the Western Pacific, the modification of temperature anomalies remains 

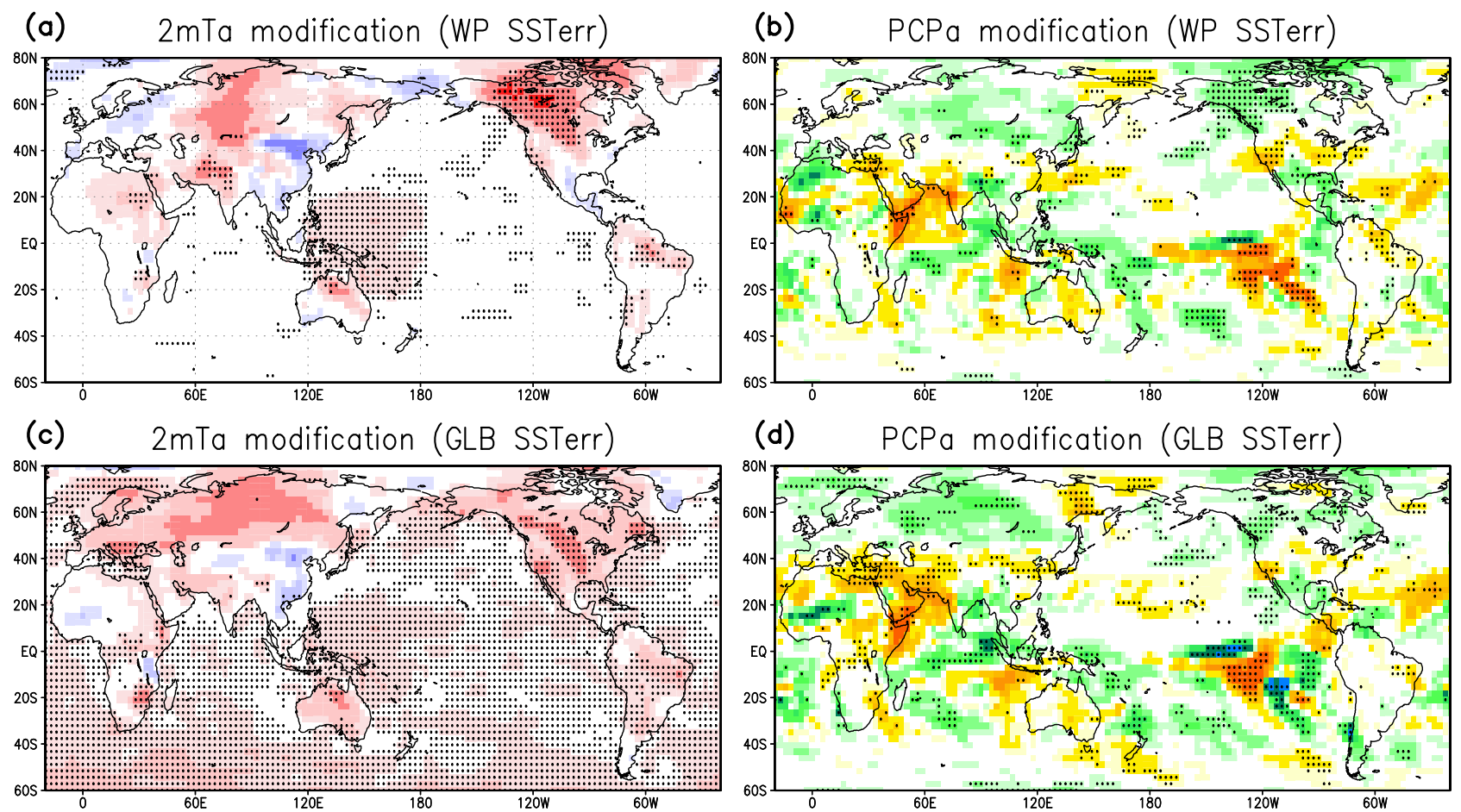

(d) PCPa modification (GLB SSTerr)
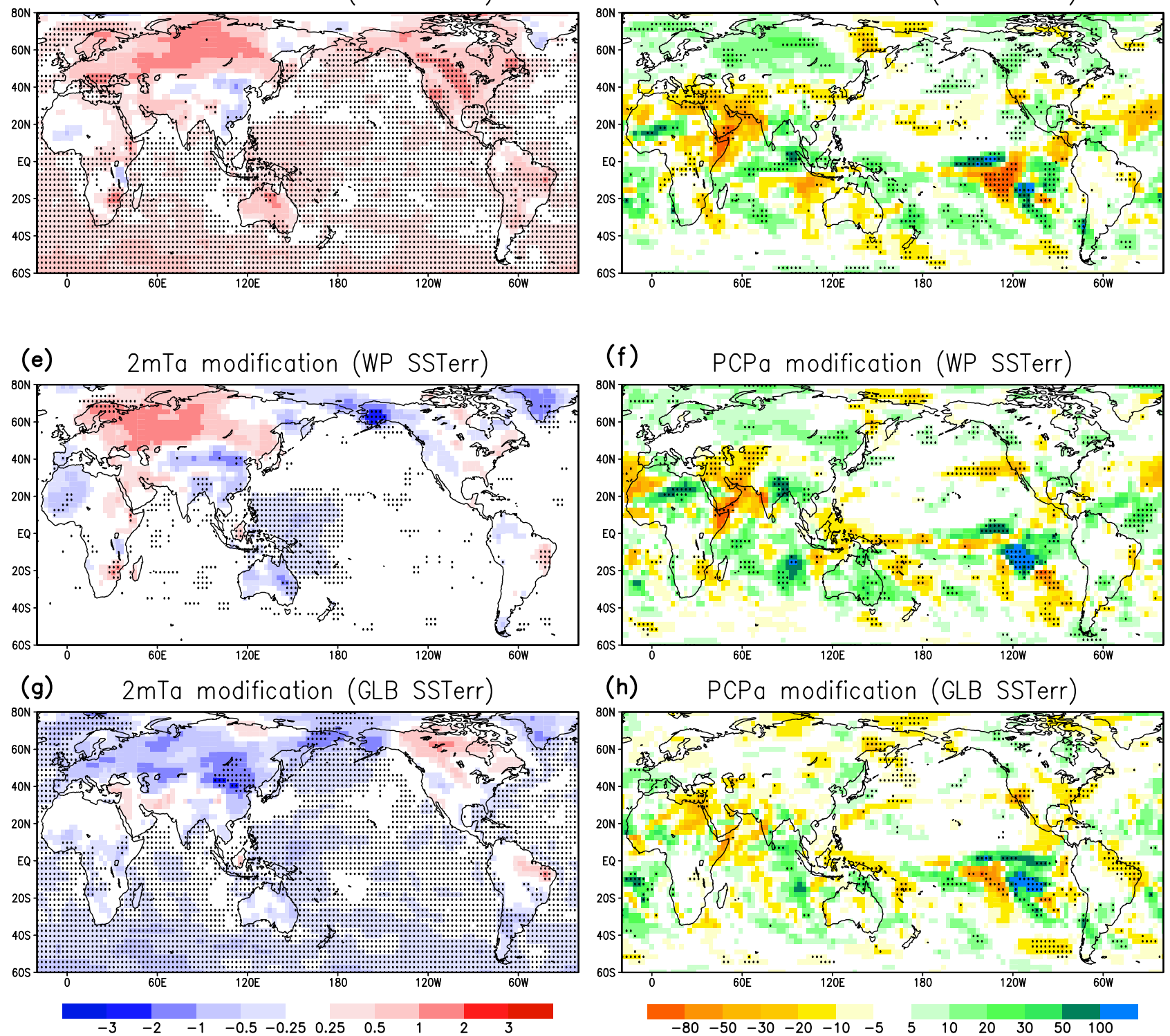

Figure 3. Modification of seasonal precipitation (\%-normal) and temperature $\left({ }^{\circ} \mathrm{C}\right)$ anomalies, due to (a-d) addition and $(\mathrm{e}-\mathrm{h})$ subtraction of estimated error in observed SSTs from JFM 1983. Sensitivity to error imposed over western Pacific region (Figures 3a, 3b, 3e, and 3f). Sensitivity to error imposed over global oceans (Figures 3c, 3d, 3g, and 3h). Local significance is assessed by t-test of the AGCM ensemble. Stippling indicates exceedance of $95 \%$ significance. 
Table 1. Spatial Correlations of Seasonal Climate Anomaly Modifications Between Experiments That Added or Subtracted SST Errors Over the Global Oceans and Those That Considered SST Errors Only Over Western Pacific Region ${ }^{\mathrm{a}}$

\begin{tabular}{|c|c|c|c|c|}
\hline & \multicolumn{2}{|c|}{$\begin{array}{c}2 \mathrm{~m} \text { Temperature } \\
\text { Anomalies }\end{array}$} & \multicolumn{2}{|c|}{ Precipitation Anomalies } \\
\hline & - Error & + Error & - Error & + Error \\
\hline \multicolumn{5}{|c|}{ Globe } \\
\hline 1983 & 0.08 & 0.51 & 0.56 & 0.67 \\
\hline 1986 & 0.55 & 0.30 & 0.68 & 0.36 \\
\hline 1989 & 0.60 & 0.13 & 0.38 & 0.22 \\
\hline \multicolumn{5}{|c|}{ Tropics $(23 S-23 N)$} \\
\hline 1983 & 0.47 & 0.61 & 0.58 & 0.68 \\
\hline 1986 & 0.79 & 0.18 & 0.71 & 0.37 \\
\hline 1989 & 0.16 & -0.19 & 0.37 & 0.24 \\
\hline \multicolumn{5}{|c|}{ N. America $(10 \mathrm{~N}-65 \mathrm{~N} ; 150 \mathrm{~W}-50 \mathrm{~W}$} \\
\hline 1983 & 0.04 & 0.60 & 0.46 & 0.67 \\
\hline 1986 & 0.08 & 0.58 & 0.41 & 0.24 \\
\hline 1989 & 0.85 & 0.18 & 0.45 & -0.06 \\
\hline
\end{tabular}

${ }^{a}$ Correlation values in bold type exceed $95 \%$ significance, based on effective spatial degrees of freedom (Globe: $\operatorname{dof}=16, \mathrm{r}_{95}=0.41$; Tropics: dof $=11, \mathrm{r}_{95}=0.50 ; \mathrm{N}$. America: dof $\left.=3, \mathrm{r}_{95}=0.9\right)$ [Bretherton et al., 1999].

similar in magnitude, and to a lesser degree spatial structure, to that from global error (Figures 3a and 3e). Significant regions are typically more pronounced in magnitude and spatial extent in the Western Pacific experiments, particularly over North America. This implies that errors in SSTs in the western Pacific dominate the perturbation response, and that the atmospheric circulation is quite sensitive to SST errors there, at least as evidenced in this AGCM. The modifications due only to the SST errors in the western Pacific in many cases bear a strong spatial resemblance to those imposed by the global SST error (Table 1).

[14] The modification patterns of the terrestrial climate anomalies are not symmetric with respect to the sign of the imposed error. Thus, uncertainty in estimates of warm pool regions may mislead diagnosis of even climatological biases in AGCMs. A significant non-linear response to tropical Pacific SSTs has been noted previously, although those studies typically focused on larger SST differences like between El Niño and La Niña events, which differ in both average magnitude and spatial details. Hoerling et al. [2001] suggest that non-linearity in the tropical rainfall response is related to an SST threshold for convection, such that the response increases linearly for warm SST anomalies but not for cold. Such a conclusion would be difficult to support here, however, given the small magnitude of the differences in anomalies imposed in these experiments. This matter requires further investigation.

[15] These results indicate that western Pacific warm pool anomalies exert considerable influence on the global climate, as found previously in other idealized AGCM experiments [e.g., Barsugli and Sardeshmukh, 2002]. The climate anomalies associated with western Pacific SST anomalies rival those forced by SST variability in other parts of the tropical oceans, where larger interannual variability is found

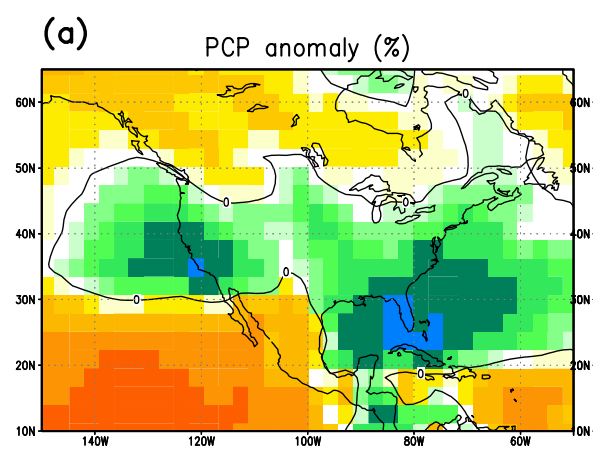

(b)

(c) Modification (-SSTerr)

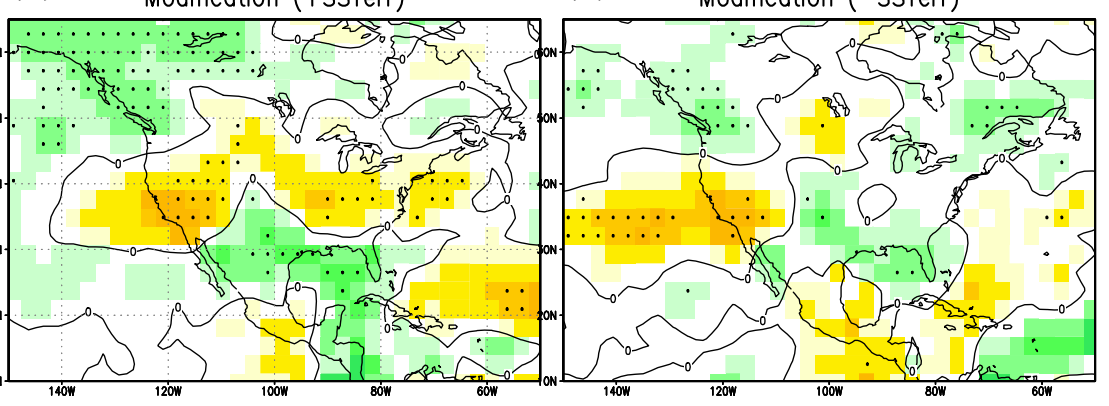

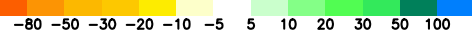

(d) $2 \mathrm{~m}$ Temperature (deg. C)

(e)

$(f)$

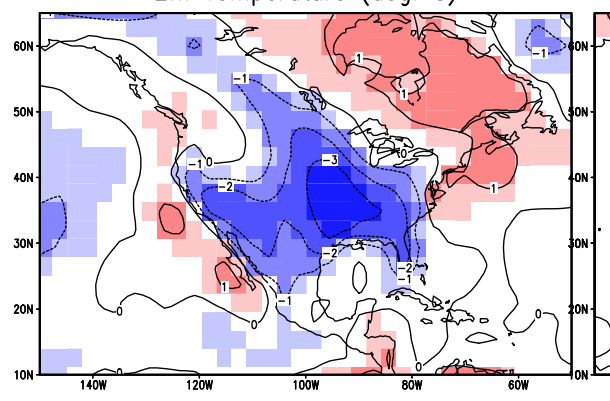

Modification (+SSTerr)

Modification (-SSTerr)

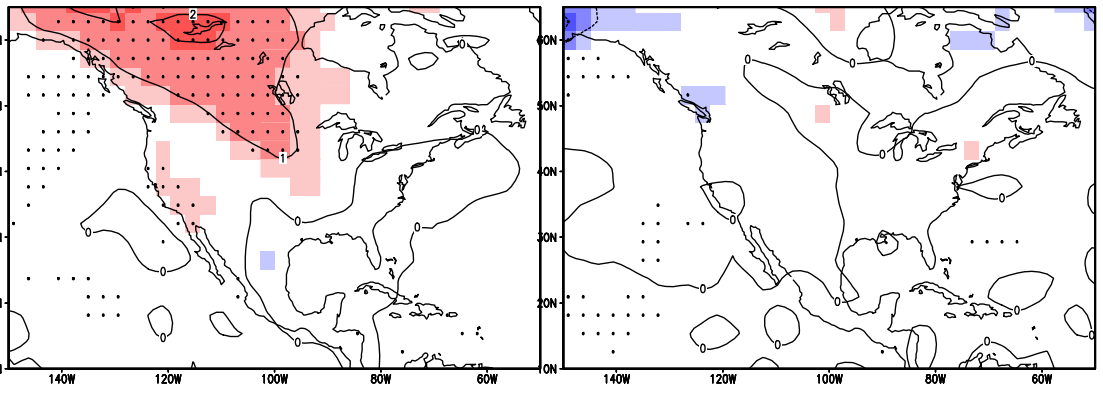

Figure 4. Ensemble mean seasonal climate anomalies, due to prescribed SSTs from JFM 1983. (a) Precipitation (\%normal) and (d) $2 \mathrm{~m}$ temperature anomalies $\left({ }^{\circ} \mathrm{C}\right.$ ) with anomalies relative to 20 -year climatology; (b and e) modification of the anomalies due to positive SST error over the western Pacific; and, (c and f) modification of the anomalies due to negative SST error. Local significance of the modified anomalies is assessed by t-test of the AGCM ensemble. Stippling indicates exceedance of $95 \%$ significance. 
and where SSTs are better sampled by in situ and satellite measurements. Over North America the AGCM-simulated air temperature and precipitation anomalies are substantially modified by the SST errors in the western Pacific (Figure 4). In some cases, the climate anomaly modifications associated with the SST errors are of comparable magnitude to those forced by the 'true SST anomalies'.

[16] The differences in AGCM response due to SST uncertainties have several implications for seasonal climate prediction. First, the added climate uncertainty makes estimation of potential predictability of the climate less robust. Potential predictability quantifies the predictability of the climate if the SSTs could be predicted 'perfectly'. Potential predictability is never perfect because of the sensitivity to initial conditions from the internal chaotic dynamics of the atmosphere leading to inherent uncertainty in the seasonal climate. Uncertainty in observed SSTs presents another source of uncertainty in the climate and thus lowers estimates of potential predictability. The second implication applies to the probabilistic calibration of models, and is related to the first. Probabilistic calibration of models requires an ability to estimate the realism of signalto-noise characteristics, where the signal is the ensemble mean response to imposed boundary conditions and the noise is the average ensemble member deviation from the mean arising from the atmospheric internal dynamics. If the SST uncertainties are not treated rigorously, the probabilistic calibration of models is likely to be sub-optimal. The availability of SST uncertainty estimates at least allows for consideration of their impact, and so allows for reliable probabilistic forecasts, even if the forecast probabilities may not as sharp as they would with more accurate measurements of SSTs. This is less important for CGCMs, where the SST uncertainty is automatically captured in the ensemble model response, and the probabilities can be calibrated directly. A third implication, concerns conclusions from attribution exercises that diagnose the causes of regional interannual climate variability, which will be degraded by inaccurate SST estimates [Hoerling and Kumar, 1997]. Given that the errors in the western Pacific are of similar magnitude to the estimated interannual variance, attribution studies of seasonal-to-interannual climate variability that seek to identify the contributions of regional SST anomalies to regional climate anomalies will suffer from an inability to estimate the relative impact from SSTs in the western Pacific. There are further implications for the real-time climate predictions as these regions are not well predicted by dynamical or statistical models.

[17] The results presented here and their implications for climate prediction and attribution efforts point to the need for accurate estimation, and prediction, of SST anomalies, particularly in the Western Pacific.

\section{Conclusions}

[18] The main conclusions from this study are summarized as:

[19] 1. Estimated errors in monthly mean SSTs within the tropics are most notable in convective regions, such as the western Pacific and Indian Oceans.
[20] 2. SST errors lead to a discernible modification of both tropical and extra-tropical precipitation and temperature anomalies.

[21] 3. The seasonal climate, globally and specifically over North America, appears particularly sensitive to SST errors in the Western Pacific.

[22] Given that the largest uncertainties in SST observations exist near warm pool regions, the variability of which influences global climate variability, this study suggests that the global ocean observing system should invest more insitu observations for these areas. This study also suggests that more explicit treatment of uncertainties be considered in climate prediction and attribution studies, such as

[23] 1. The skill of SST predictions should be considered relative to such an upper limit and explicitly account for the uncertainties in the verification data, in addition to conventional presentation of prediction skill;

[24] 2. Seasonal climate prediction research should consider how to quantify the reduction in potential predictability arising from imperfect specification of SSTs in AGCM simulations;

[25] 3. Attribution studies of seasonal climate anomalies should conduct additional sensitivity studies considering SST errors, when variability of warm pool regions is implicated.

[26] Acknowledgments. The authors acknowledge helpful suggestions from Tom Smith and Simon Mason. We appreciate Xiaofeng Gong's help in running and processing the AGCM experiments. This work was funded under grant NA03OAR4320179 from NOAA's Office of Climate Observations and cooperative agreement NA05OAR4311004 between Columbia University and the National Oceanic and Atmospheric Association.

\section{References}

Barsugli, J. J., and P. D. Sardeshmukh (2002), Global atmospheric sensitivity to tropical SST anomalies throughout the Indo-Pacific basin, J. Clim., 15, 3427-3442.

Bretherton, C. S., M. Widmann, V. P. Dymnikov, J. M. Wallace, and I. Bladé (1999), The effective number of spatial degrees of freedom of a time-varying field, J. Clim., 12, 1990-2009.

Hoerling, M. P., and A. Kumar (1997), Origins of extreme climate states during the 1982-83 ENSO winter, J. Clim., 10, 2859-2870.

Hoerling, M. P., A. Kumar, and T. Xu (2001), Robustness of the nonlinear climate response to ENSO's extreme phases, J. Clim., 14, 1277-1293.

Office of Climate Observations (2008), Program plan for building a sustained ocean observing system for climate, report, 63 pp., NOAA, Silver Spring, Md.

Roeckner, E., et al. (1996), The atmospheric general circulation model ECHAM4: Model description and simulation of present-day climate, MPI Rep. 218, 90 pp., Max Planck Inst. for Meteorol., Hamburg, Germany.

Smith, T. M., and R. W. Reynolds (2004), Improved extended reconstruction of SST (1854-1997), J. Clim., 17, 2466-2477.

Smith, T. M., and R. W. Reynolds (2005), A global merged land air and sea surface temperature reconstruction based on historical observations (1880-1997), J. Clim., 18, 2021-2036.

D. G. DeWitt and L. Goddard, International Research Institute for Climate and Society, Earth Institute at Columbia University, 22 Monell Building, 61 Route 9W, Palisades, NY 10964, USA. (goddard@iri.columbia.edu)

R. W. Reynolds, National Climate Data Center, NOAA, 5200 Auth Road, Room 807, Camp Springs, MD 20746, USA. 